Article

\title{
Lempel-Ziv Complexity of Photonic Quasicrystals
}

\author{
Juan J. Monzón ${ }^{1}$, Angel Felipe ${ }^{2}$ and Luis L. Sánchez-Soto ${ }^{1,3, *}$ \\ 1 Departamento de Óptica, Facultad de Física, Universidad Complutense, 28040 Madrid, Spain; \\ jjmonzon@opt.ucm.es \\ 2 Departamento de Estadística e Investigación Operativa, Facultad de Matemáticas, \\ Universidad Complutense, 28040 Madrid, Spain; felipe@mat.ucm.es \\ 3 Max Planck Institut für die Physik des Lichts, Staudtstraße 2, 91058 Erlangen, Germany \\ * Correspondence: lsanchez@fis.ucm.es
}

Academic Editor: Helmut Cölfen

Received: 27 April 2017; Accepted: 21 June 2017; Published: 23 June 2017

\begin{abstract}
The properties of one-dimensional photonic quasicrystals ultimately rely on their nontrivial long-range order, a hallmark that can be quantified in many ways depending on the specific aspects to be studied. Here, we assess the quasicrystal structural features in terms of the Lempel-Ziv complexity. This is an easily calculable quantity that has proven to be useful for describing patterns in a variety of systems. One feature of great practical relevance is that it provides a reliable measure of how hard it is to create the structure. Using the generalized Fibonacci quasicrystals as our thread, we give analytical fitting formulas for the dependence of the optical response with the complexity.
\end{abstract}

Keywords: quasicrystals; photonic crystals; photonic bandgap materials

\section{Introduction}

The spatial modulation of the properties of a medium enforces drastic alterations in light propagation. There are two extreme instances of this: when the spatial profile is periodic and when it is fully random. In the first case, the structure is called a photonic crystal [1,2], underlining the strong similarities between the distinctive features of light in these structures and those of electrons in solids. Bragg mirrors, consisting of alternating low- and high-index layers, constitute the simplest example of one-dimensional photonic crystals [3,4]: the name stems from the presence of photonic band gaps; i.e., ranges of frequency in which strong reflection occurs for all angles of incidence and all polarizations [5-9].

The converse case of random media has also attracted attention because it lies in the realm of some intriguing effects, such as Anderson localization [10,11], coherent backscattering [12], and the optical Hall effect [13].

Deterministic aperiodic spatial patterns, dubbed photonic quasicrystals [14-21], display optical responses that cannot be found in either periodic or random systems. Among other issues, they include a self-similar energy spectrum [22,23], a pseudo-bandgap of forbidden frequencies [24-26], and critically localized states $[27,28]$ whose wave functions are distinguished by power law asymptotes and self-similarity [29-31]. In fact, many of these traits have been found to be useful [32,33].

The first illustration of an aperiodic lattice possessing long-range order was a one-dimensional structure assembled according to the Fibonacci sequence [34-36]. Subsequently, a wealth of other photonic quasicrystals have been conceived, the most outstanding ones being Thue-Morse [37-39] and Cantor quasicrystals [40,41].

In this work, we focus on the so-called generalized Fibonacci quasicrystals, a topic that has been growing over the last few years [42-56]. Besides, it is underpinned by remarkable mathematical properties [57], which confirms that deterministic aperiodic nanostructures are a highly interdisciplinary area of research. 
Generally, one can classify deterministic aperiodic systems by their Fourier spectrum. Yet, unveiling the relations between this spectrum and the physical properties is a problematic task. As these lattices can be conveniently generated by substitution sequences, an alternative route is to look at the complexity of the resulting arrangement. There are many measures of complexity available [58], each one with its pros and cons. However, in recent years the Lempel-Ziv (LZ) complexity stands out because it is relatively easy to compute and gives results of very direct interpretation [59]. Indeed, it is related to the number of distinct patterns and the rate of their occurrence along a given structure and this is a rough indication of how hard is to create it.

One direct way of comparing the optical properties of different lattices is to measure, e.g., the reflectance. In this work, we find closed expressions relating the reflectance with the complexity. We thereby gain further insight into the relation between structural and spectral properties that governs the optical behavior of one-dimensional quasicrystals with controllable degree of aperiodic order.

\section{Generalized Fibonacci Quasicrystals}

Let us first review some basic notions that are important for the discussion that follows. A word (also called a sequence) is an ordered list of letters, which are elements of a finite alphabet. We shall be concerned with a two-letter alphabet, denoted by $\{L, H\}$, but alphabets can be of any size. In physical realizations, each letter corresponds to a different type of building block (e.g., dielectric layers, atoms, etc.).

A straightforward way to generate deterministic aperiodic words is using symbolic substitutions [60,61]. A specific substitution rule replaces each letter in the alphabet by a finite word:

$$
L \mapsto \varphi_{1}(L, H), \quad H \mapsto \varphi_{2}(L, H),
$$

$\varphi_{1}$ and $\varphi_{2}$ being any string of $L$ and $H$. One must start from a given letter, which is called a seed.

More specifically, we are interested in the generalized Fibonacci sequences $\operatorname{FS}(h, \ell)$, which are generated by the inflation rule

$$
L \mapsto H, \quad H \mapsto H^{h} L^{\ell},
$$

where $h$ and $\ell$ are arbitrary positive integers and we adopt the convention that the seed is $L$.

The words $\left\{W_{\alpha}\right\}$ of $\mathrm{FS}(h, \ell)$ can be alternatively defined by the recursive scheme

$$
W_{\alpha+1}=W_{\alpha}^{h} W_{\alpha-1}^{\ell},
$$

with $W_{0}=L$ and $W_{1}=H$. Here, the integer $\alpha$ labels the corresponding step in the iteration, which is also known as the generation.

The length (i.e., the total number of letters $L$ and $H$ ) of the word $W_{\alpha}$ is denoted by $w_{\alpha}$ and satisfies the relation

$$
w_{\alpha+1}=h w_{\alpha}+\ell w_{\alpha-1} .
$$

In the limit of an infinite sequence, we have that

$$
\lim _{\alpha \rightarrow \infty} \frac{w_{\alpha}}{w_{\alpha-1}} \equiv \sigma(h, \ell)=\frac{1}{2}\left(h+\sqrt{h^{2}+4 \ell}\right) .
$$

For $\ell=1$, the resulting sequences fulfill

$$
\sigma(h, 1)=h+\frac{h}{h+\frac{h}{h+\ddots}} .
$$


If we denote the continued fraction appearing in this equation as $[\bar{h}]=[h, h, h, \ldots]$, we can write

$$
\sigma(1,1)=\frac{1}{2}(1+\sqrt{5}) \equiv \Phi=[\overline{1}], \quad \sigma(2,1)=1+\sqrt{2}=[\overline{2}], \quad \sigma(3,1)=\frac{1}{2}(3+\sqrt{13})=[\overline{3}],
$$

and, in general, $\sigma(h, 1)=[\bar{h}]$. For $\sigma(1,1)$ (i.e., the standard Fibonacci sequence), we get the golden mean $\Phi$, whereas $\sigma(1,2)$ gives the silver mean and $\sigma(3,1)$ the bronze mean. This family generalizes in quite a natural way the golden ratio [62] and will be designated here, by obvious reasons, as the Olympic-metal family. Among all these metallic means, the one that converges slowest is the golden mean, since its denominators are the smallest possible. This corresponds to the traditional statement that the golden mean $\Phi$ is the most irrational of all irrational numbers [57].

On the other hand, when we fix $h=1$, the sequences satisfy

$$
\sigma(1,2)=2, \quad \sigma(1,3)=[2, \overline{3}], \quad \sigma(1,4)=[2, \overline{1,1,3}], \quad \sigma(1,5)=[2, \overline{1,3}], \quad \sigma(1,6)=3,
$$

and so on. The complete FS $(1, h)$ series will be termed as the non-Olympic-metal family. However, notice that there are instances in this series for which $\sigma(1, h)$ becomes an integer and we could expect a different behavior. To take this into due consideration, we will study here the first two representatives of this family: $\mathrm{FS}(1,2)$; the copper mean, and FS(1,3); the nickel mean.

In an optical implementation of the generalized Fibonacci sequences FS $(h, \ell)$, the letters in the alphabet $\{L, H\}$ are realized as layers made of materials with refractive indices $\left(n_{L}, n_{H}\right)$ and thicknesses $\left(d_{L}, d_{H}\right)$, respectively. The material $L$ has a low refractive index, while $H$ is of a high refractive index, which justifies the notation. An illustration of the resulting systems is presented in Figure 1.
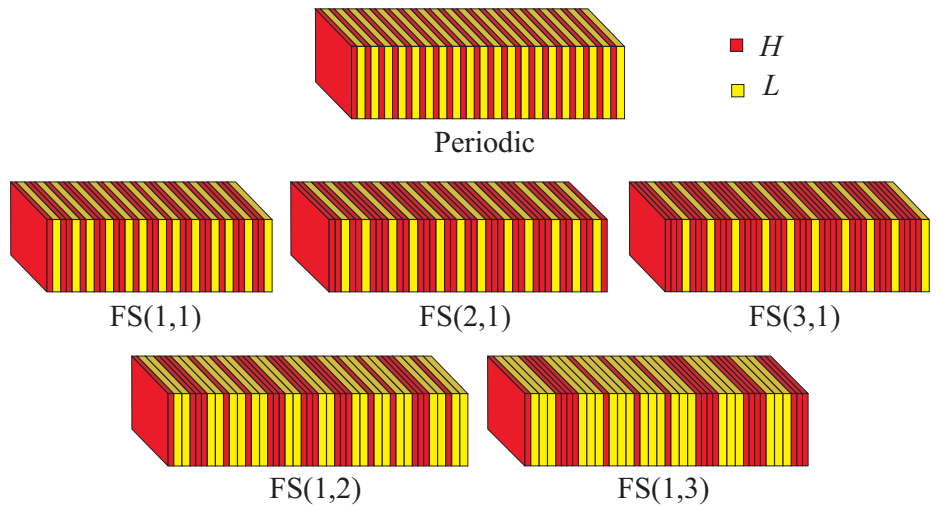

Figure 1. Illustrations of the different quasiperiodic chains considered in this work. In the top panel, we have the periodic case (40 letters). In the mid panel, the Olympic-metal family Fibonacci sequence (FS) $(h, 1)$, with (from left to right) $h=1$ (golden mean, 34 letters), $h=2$ (silver mean, 41 letters), and $h=3$ (bronze mean, 43 letters). In the bottom panel, the non-Olympic-metal family FS $(1, \ell)$, with $\ell=2$ (copper mean, 43 letters) and $\ell=3$ (nickel mean, 40 letters).

The optical response of these structures can be efficiently computed using the transfer-matrix technique [63-65]. The $\alpha$ th word of $\mathrm{FS}(h, \ell)$ has the associated transfer matrix

$$
\mathrm{M}_{\alpha+1}=\mathrm{M}_{\alpha}^{h} \mathrm{M}_{\alpha-1}^{\ell},
$$

starting from $M_{0}=M_{L}$ and $M_{1}=M_{H}$, which are the basic matrices for each layer. Once $M_{\alpha}$ is known, the transmittance

$$
\mathcal{T}_{\alpha}=\frac{4}{\left|\mathrm{M}_{\alpha}\right|^{2}+2}
$$


where $\left|\mathrm{M}_{\alpha}\right|^{2}=\sum_{i j}\left|m_{i j}\right|^{2}$ (the sum of the absolute squares of the matrix elements) is the (Frobenius) norm of $\mathrm{M}_{\alpha}$. Note that $\mathcal{T}_{\alpha}$ will depend on the wavelength, incidence angle and polarization of the incident radiation.

\section{Spectral Measures of Generalized Fibonacci Quasicrystals}

To each rule (1) we associate a substitution matrix $T$, defined as

$$
\mathbf{T}=\left(\begin{array}{cc}
\left|\varphi_{1}(L, H)\right|_{L} & \left|\varphi_{2}(L, H)\right|_{L} \\
\left|\varphi_{1}(L, H)\right|_{H} & \left|\varphi_{2}(L, H)\right|_{H}
\end{array}\right),
$$

where $|\cdot|_{L, H}$ is the number of letters $L$ (resp. $H$ ). This matrix does not depend on the precise form of the substitutions, but only on the number of letters $L$ or $H$.

The eigenvalues of $\mathrm{T}$ contain a lot of information. Actually, as discovered by Bombieri and Taylor [66,67], if the spectrum of $T$ contains a Pisot number as an eigenvalue, the sequence is quasiperiodic; otherwise it is not (and then is purely aperiodic). We recall that a Pisot number is a positive algebraic number (i.e., a number that is a solution of an algebraic equation) greater than one, all of whose conjugate elements (the other solutions of the defining algebraic equation) have modulus less than unity [68].

For the family $\mathrm{FS}(h, \ell)$, we have

$$
\mathrm{T}_{h, \ell}=\left(\begin{array}{cc}
h & 1 \\
\ell & 0
\end{array}\right)
$$

whose eigenvalues are

$$
\tau_{h, \ell}^{( \pm)}=\frac{1}{2}\left(h \pm \sqrt{h^{2}+4 \ell}\right) .
$$

Incidentally, the largest eigenvalue $\tau_{h, \ell}^{(+)}$, which is often known as the Perron-Frobenius eigenvalue [69], coincides with the ratio $\sigma(h, \ell)$.

The eigenvalues $\tau_{h, 1}$ are Pisot numbers, so all the sequences in the Olympic-metal family $\mathrm{FS}(h, 1)$ are quasiperiodic. In contradistinction, $\tau_{1, \ell}$ are not Pisot numbers and the corresponding non-Olympic-metal systems FS $(1, \ell)$, are aperiodic.

The main differences between these two situations can be appreciated by considering the nature of their Fourier spectra [70]. For a specific word of length $n$, the discrete Fourier transform reads

$$
\hat{\mathrm{W}}_{n}(k)=\frac{1}{\sqrt{n}} \sum_{j=0}^{n-1} W(j) \exp \left(-\frac{2 \pi \mathrm{i} k j}{n}\right), \quad k=1,2 \ldots, n,
$$

where $W(j)$ is a numerical array obtained from the word by assigning to each letter of the alphabet a fixed number. This assignment is otherwise arbitrary and does not change our conclusions. As a consequence, one could, e.g., use $L \mapsto-1$ and $H \mapsto 1$. The structure factor (or power spectrum) is [71]

$$
F_{n}(k)=\left|\hat{W}_{n}(k)\right|^{2} .
$$

From a mathematical perspective, the only well-established concept attached to the Fourier spectrum is its spectral measure. If $d v_{n}(k)=F_{n}(k) d k$, we will be concerned with the limit $d v(k)=\lim _{n \rightarrow \infty} d v_{n}(k)$, which corresponds to an infinite structure and a continuous variable $k$. Like any positive measure, $d v(k)$ can be uniquely decomposed as

$$
d v(k)=d v_{\mathrm{pp}}(k)+d v_{\mathrm{ac}}(k)+d v_{\mathrm{sc}}(k),
$$

i.e., into its pure point, absolutely continuous and singular continuous parts [33]. 
These spectral measures are often identified with the characteristics of the transmission spectra of the structures. The pure point part refers to the presence of Bragg peaks; the absolute continuous part is a differentiable function (diffuse scattering), while the singular continuous part is neither continuous nor does it have Bragg peaks; it shows broad peaks, which are never isolated and, with increasing resolution, split again into further broad peaks.

For example, in random media in the diffusive regime, uncorrelated disorder gives rise to an absolutely continuous diffraction measure. In contradistinction, in the regime of Anderson localization, where exponentially localized states occur at discrete resonant frequencies, the energy spectrum is pure point. On the other hand, periodic structures exhibit sharp diffraction Bragg peaks (i.e., a pure-point measure) due to their long-range periodic order, but they may also support continuous energy bands.

The Fibonacci lattice has a pure-point spectrum with a countable set of peaks at incommensurate intervals. The chief example of a deterministic sequence with a singular continuous spectrum is the Thue-Morse sequence [39].

In Figure 2 we plot the power spectrum $F_{n}(k)$ for the Olympic- and non-Olympic-metal families. The former exhibit $\delta$-like Bragg peaks that can be properly labeled in terms of the eigenvalues $\tau_{h, 1}$ as

$$
k_{m_{1} m_{2}}=\frac{2 \pi}{\Lambda_{0}} m_{1} \tau_{h, 1}^{m_{2}}
$$

with $m_{1}$ and $m_{2}$ integers and $\Lambda_{0}$ being a suitable average period of the structure. We can verify the existence of incommensurate intervals between peaks, confirming the quasiperiodicity of these arrangements. Moreover, the gap-labelling theorem [72] relates the position of the peaks in Equation (17) with the location of the gaps in the energy spectra of the elementary excitations supported by the structure.
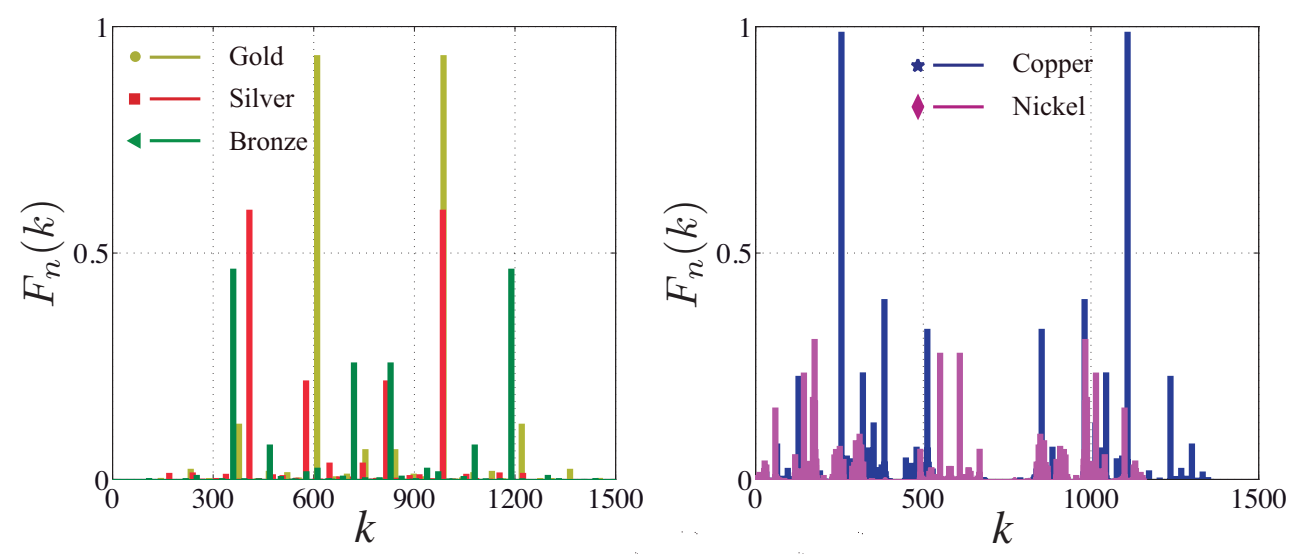

Figure 2. Normalized power spectrum for words up to 1500 letters for different generalized Fibonacci sequences. In the left panel, for the Olympic-metal family $\mathrm{FS}(h, 1)$, with $h=1,2$, and 3 . In the right panel, for the non-Olympic-metal sequences FS $(1,2)$ and FS $(1,3)$.

For non-Olympic metals, the global structure looks blurred. Individual Bragg peaks are not separated by well-defined intervals, but tend to cluster, forming "broad bands". The strength of the dominant peaks is considerably bigger for the copper lattice, which suggests that the nickel lattice is more disordered than the copper one. A careful analysis of these issues [45] shows that these spectra are multifractal and their Fourier-spectral measures are singular continuous ones.

\section{Lempel-Ziv Complexity}

The Fourier power spectrum conveys complete information about the system. However, while the qualitative features can be understood at a simple glance, extracting useful quantitative data is by no 
means obvious. As heralded in the Introduction, to bypass this drawback, we propose to assess the long-range order in terms of the closely related concept of complexity [73].

Of course, any definition of complexity is beholden to the perspective brought to bear upon it. In a broad sense, the complexity lies in the difficulty faced in describing system characteristics. For example, the two cases discussed in the Introduction, namely the periodic and the random spatial profiles, are extreme examples of simple models and therefore systems with zero complexity.

A perfect crystal is completely ordered and the components are arranged following stringent rules of symmetry. A small piece of information is enough to describe the perfect crystal and the information stored in this system can be considered minimal. On the other hand, the random profile is completely disordered: the system can be found in any of its accessible states with the same probability and it has therefore a maximum information. These two simple systems are extrema in the scale of order and information. We thus conclude that a sensible definition of complexity must not be made in terms of just order or information.

The LZ complexity [74] and its variants are popular measures for characterizing the randomness of a sequence. Indeed, in a way, it gives a clear indication of how hard is it to create the sequence, so it is a sensible quantity from an experimental viewpoint. For a word $W_{n}=\left\{x_{1} x_{2} \ldots x_{n}\right\}$ of length $n \mathrm{t}\left(x_{i} \in\{L, H\}, 1 \leq i \leq n\right)$, a procedure that partitions $W_{n}$ into non-overlapping blocks is called a parsing. A block starting at position $i$ and ending at position $j$ of $W_{n}$ is often called a phrase $W_{n}(i, j)$. The set of phrases generated by a parsing of $W_{n}$ is denoted by $P W_{n}$ and the number of phrases by $c\left(W_{n}\right)$.

Assume that a word $W_{n}$ has been parsed up to position $i$, so that $P W_{n}(1, i)$ is the set of phrases generated so far. According to the original LZ procedure, the next phrase $W_{n}(i+1, j)$ will be the first block which is not yet an element of $P W_{n}(1, i)$. As an illustration, the string LLHHLLHLHLHLLHHH will be parsed as $L|L H| H L|L H L| H L H L L \mid H H H$.

The LZ complexity $C\left(W_{n}\right)$ is defined as

$$
C\left(W_{n}\right)=\frac{1}{n} c\left(W_{n}\right)\left[\log _{2} c\left(W_{n}\right)+1\right] .
$$

For a random word $W_{n}$, it can be shown that $\lim _{n \rightarrow \infty} C\left(W_{n}\right)$ gives the Shannon entropy rate. As a consequence, the LZ complexity also quantifies the average amount of information in the Shannon sense. Note that the normalization in Equation (18) guarantees that $0 \leq C\left(W_{n}\right) \leq 1$ only in the asymptotic limit; for finite $n$, we can find instances for which $C\left(W_{n}\right)$ is greater than one. Since $C$ is based on the study of recurrence of patterns in a symbolic sequence, this approach provides a tool for the analysis of complex sequences.

\section{Complexity of Generalized Fibonacci Quasicrystals}

Next, we analyze the different response of the metal families discussed thus far as a function of their LZ complexity. For that purpose, we concentrate on a definite quantity: the transmittance, which in the presence of a pseudo-bandgap tends to zero.

To keep irrelevant details apart, we deal with normal incidence at a definite wavelength. For definiteness, for the practical realization of the layers, the materials are chosen to be cryolite $\left(\mathrm{Na}_{3} \mathrm{AlF}_{6}\right)$ and zinc selenide (ZnSe), with refractive indices $n_{L}=1.34$ and $n_{H}=2.568$, respectively, at $\lambda=0.65 \mu \mathrm{m}$. This is a standard choice and, although it is a simple example, it allows one to work out easily the details of the method, which can be immediately extended to other media and spectral regions.

To allow for a fair benchmarking, we have to set a criterion that fixes the thicknesses in a consistent manner. To this end, we impose that, for each metal, the average transmittance

$$
\overline{\mathcal{T}}=\frac{1}{\alpha_{\max }} \sum_{\alpha=1}^{\alpha_{\max }} \mathcal{T}_{\alpha}
$$


is minimal. In $\overline{\mathcal{T}}$, we choose a cutoff of the number of generations $\alpha_{\max }$ such that for $\alpha>\alpha_{\max }$, $-\ln \mathcal{T}_{\alpha}>200$, and so they can be safely ignored. This is precisely the range plotted in Figure 3. This uniquely determines $d_{H}$ and $d_{L}$ for the metal family under consideration.

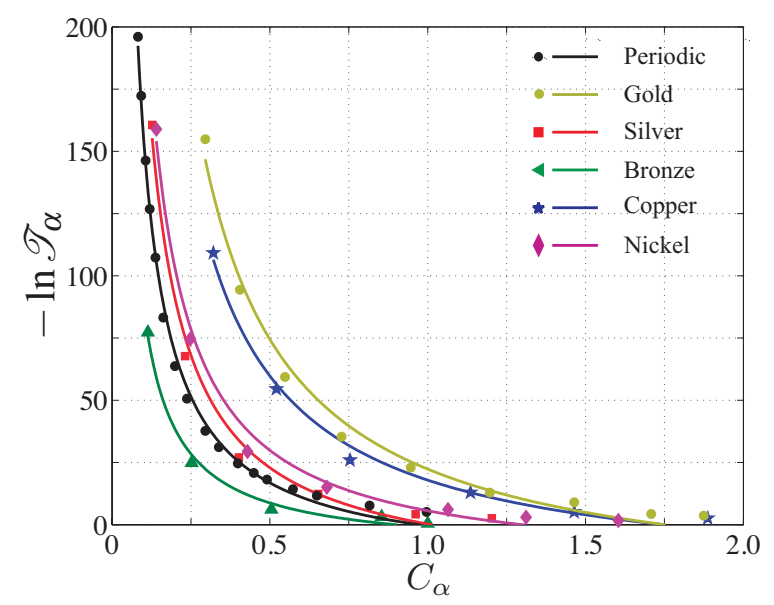

Figure 3. Behavior of the transmittance as a function of the LZ complexity for the periodic system and the generalized Fibonacci families indicated in the inset. We consider generations up to 1600 letters.

To obtain these optimal values, the two thicknesses are varied independently from $0.02 \mu \mathrm{m}$ to $0.30 \mu \mathrm{m}$. This range is in turn subdivided into 50 even intervals, so all in all we get a set of $50 \times 50$ equal rectangles. The center of each rectangle is chosen as an initial guess and we seek a best point by using a random permutation of the two thicknesses and an iterative search with fixed (positive or negative) increment. In the next step of optimization, we apply a quasi-Newton algorithm to improve the points of the previous exploration.

We have also included the periodic crystal $[L H]^{n}$, for which the optimal solution is known to be

$$
n_{L} \frac{d_{L}}{\lambda}=\frac{1}{4}, \quad n_{H} \frac{d_{H}}{\lambda}=\frac{1}{4} .
$$

The resulting values, expressed in the dimensionless form $n d / \lambda$, are given in Table 1 . In general, all the families present $n_{L} d_{L} / \lambda$ lesser than 0.25 , except the bronze. The values of $n_{H} d_{H} / \lambda$ are close to 0.25 , except for the gold and silver.

Table 1. Thicknesses yielding minimum average transmittance over all the generations in the generalized Fibonacci quasicrystals indicated in the first column.

\begin{tabular}{cccc}
\hline System & Metal & $n_{L} d_{L} / \lambda$ & $n_{H} d_{H} / \lambda$ \\
\hline Periodic & - & 0.2500 & 0.2500 \\
FS(1,1) & Gold & 0.2251 & 0.3417 \\
FS(2,1) & Silver & 0.2311 & 0.3809 \\
FS(3,1) & Bronze & 0.3416 & 0.2459 \\
FS(1,2) & Copper & 0.1249 & 0.2501 \\
FS $(1,3)$ & Nickel & 0.2451 & 0.2277 \\
\hline
\end{tabular}

In Figure 3 we plot the logarithm of the transmittance $-\ln \mathcal{T}_{\alpha}$ for the different generalized Fibonacci quasicrystals as a function of the corresponding LZ complexity. This LZ complexity has been computed using an efficient implementation in Matlab [75]. The range of $-\ln \mathcal{T}_{\alpha}$ mentioned before limits the number of letters to 1600 .

Let us look at the complexity required to obtain a given transmittance: in the case of the Olympic metals, gold is the more complex, followed by silver and bronze; for the non-Olympic family, copper is 
the more complex, followed by nickel. So, they are arranged in their "natural" order according to their definition in the series $\mathrm{FS}(h, \ell)$. However, when both families are intercompared, the relative sequence does not follow a definite pattern, which surely reflects the very different nature of their associated Fourier spectra. On the other hand, the periodic Bragg reflector requires a low complexity: only the bronze requires lesser. An intuitive explanation of these facts can be found in Figure 1: the degree of organization of the systems differs in a remarkable way. In other words, the number of effective interfaces between media $L$ and $H$ may be significantly different from the total number of layers.

All the curves can be properly fitted to the functional form

$$
-\ln \mathcal{T}_{\alpha}=a_{0}+\frac{a_{1}}{C_{\alpha}}
$$

corresponding to shifted hyperbolas. The resulting coefficients $a_{0}$ and $a_{1}$ for each family can be found in Table 2. In some cases (notably, bronze) the cloud of points is not large enough to appraise at a glance the goodness of the fit. Nonetheless, the correlation coefficients $r$ are always better than 0.99 , which confirms the validity of (21). Note also that for hypothetical values of $C_{\alpha}$ greater than 2 , this fitting would eventually lead to transmittances $\mathcal{T}_{\alpha}$ greater than one, which has no physical meaning. In the realistic range considered in the plot, the hyperbolas never intersect.

Table 2. Parameters involved in the fittings in Equations (21), (22) and (24).

\begin{tabular}{cccccccc}
\hline System & Metal & $\boldsymbol{a}_{\mathbf{0}}$ & $\boldsymbol{a}_{\mathbf{1}}$ & $\boldsymbol{b}_{\mathbf{0}}$ & $\boldsymbol{b}_{\mathbf{1}}$ & $\boldsymbol{c}_{\mathbf{0}}$ & $\boldsymbol{c}_{\mathbf{1}}$ \\
\hline Periodic & - & -17.917 & 17.301 & 0.54422 & 0.04718 & -1.82860 & 0.66697 \\
FS $(1,1)$ & Gold & -29.779 & 52.116 & 0.46257 & 0.00998 & -0.35174 & 0.41042 \\
FS $(2,1)$ & Silver & -22.297 & 22.590 & 0.71580 & 0.01821 & 0.29576 & 0.27829 \\
FS(3,1) & Bronze & -10.953 & 9.847 & 0.90351 & 0.02264 & 0.28379 & 0.16478 \\
FS $(1,2)$ & Copper & -23.922 & 41.833 & 0.44177 & 0.00980 & -1.27180 & 0.32427 \\
FS(1,3) & Nickel & -18.687 & 24.282 & 0.57597 & 0.00876 & 0.16579 & 0.14514 \\
\hline
\end{tabular}

It is interesting to check how the complexity depends on the number of letters (in our case, layers) $w_{\alpha}$ in each word $W_{\alpha}$. The results are shown in Figure 4 . The curves can be fitted to the functional form
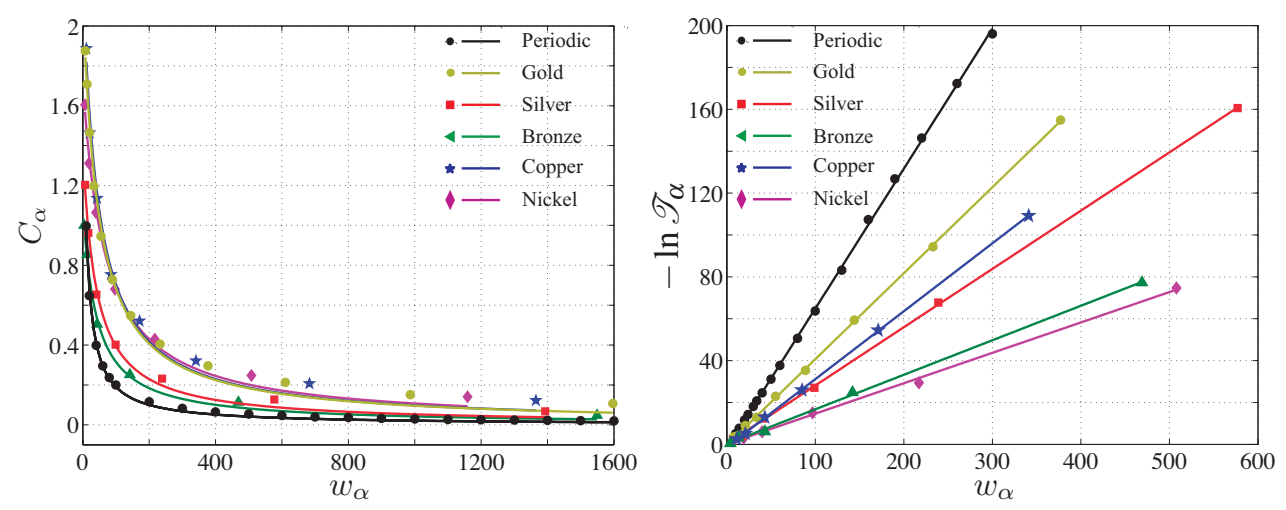

Figure 4. (Left) Lempel-Ziv (LZ) complexity versus the number of letters in the words of several generations for the periodic systems and the generalized Fibonacci quasicrystals indicated in the insets; (Right) Transmittance for the same cases as before, as a function of the number of letters $w_{\alpha}$.

$$
C_{\alpha}=\frac{1}{b_{0}+b_{1} w_{\alpha}}
$$

which is again a hyperbola, but now with $w_{\alpha}$ offset. The corresponding parameters can be found in Table 2, and the Pearson correlation coefficients are always greater than 0.99 . 
For a fixed value of $w_{\alpha}$, the periodic sequence has the lower complexity, which seems intuitively obvious. The gold and the non-Olympic series (copper and nickel) appear almost superimposed, whereas silver and bronze are in an intermediate situation.

In the limit $\alpha \rightarrow \infty$, the LZ complexity $C_{\alpha}$ tends to zero for all the sequences, which is seen directly in the Figure 4 and is evident from the fitting (22). This is consistent with the asymptotic behavior

$$
C_{\alpha} \sim \frac{\left(\ln w_{\alpha}\right)^{2}}{w_{\alpha} \ln \Phi}
$$

which has been found for the Fibonacci quasicrystals [76].

Finally, we can merge these last two results, schematized in Equations (21) and (22), to check the dependence of the transmittance with the number of layers. The final results reads

$$
-\ln \mathcal{T}_{\alpha}=c_{0}+c_{1} w_{\alpha}
$$

Again the associated parameters are given in Table 2 and they are consistent with the previous ones.

The results are presented in Figure 4. The correlation coefficients (always greater than 0.999) confirm that Equation (24) is indeed a good approximation. Note, in passing, that this implies that the reflectance approaches unity exponentially with the number of layers, as one would expect from a bandgap. The periodic stack has the biggest slope, followed by the Olympic-metal family. Unexpectedly, the copper lattice performs better than one could anticipate, while the nickel is the worst. To sum up, where the number of letters is concerned, periodicity always beats both quasiperiodic and aperiodic orders.

As a final remark, we mention that we have performed similar analysis for other quasicrystals, in particular, for generalized Thue-Morse sequences. We do not include the details here for the sake of conciseness. The conclusions are much the same as the ones presented thus far.

\section{Conclusions}

We have exploited the notion of LZ complexity to explore the performance of generalized Fibonacci sequences. This LZ complexity gives a direct estimate of how difficult it is to create the system and has a direct interpretation for the experimentalist. What we have discovered is how these sequences perform in terms of a given complexity. We think that this that might open new ways of quantifying the performance of these kinds of quasicrystals.

Acknowledgments: We thank Olivia Di Matteo for a careful reading of the manuscript. Juan Monzon and Luis Sanchez-Soto acknowledge the support of the Spanish MINECO (Grant FIS2015-67963-P).

Author Contributions: Juan Monzon conceived the research and contributed to the development of simulation methods. Angel Felipe performed the numerical simulations and optimizations. Luis Sanchez-Soto conceived the research, wrote the paper and helped with data interpretation. All authors have read and approved the final manuscript.

Conflicts of Interest: The authors declare no conflict of interest.

\section{References}

1. Joannopoulos, J.D.; Meade, R.D.; Winn, J.N.; Meade, R.D. Photonic Crystals: Molding the Flow of Light, 2nd ed.; Princeton University Press: Princeton, NJ, USA, 2008.

2. Buscha, K.; von Freymann, G.; Linden, S.; Mingaleev, S.; Tkeshelashvili, L.; Wegenerd, M. Periodic nanostructures for photonics. Phys. Rep. 2007, 444, 101-202.

3. Yeh, P. Optical Waves in Layered Media; Wiley: New York, NY, USA, 1988.

4. Lekner, J. Theory of Reflection; Kluwer: Dordrecht, The Netherlands, 1987.

5. Fink, Y.; Winn, J.N.; Fan, S.; Chen, C.; Michel, J.; Joannopoulos, J.D.; Thomas, E.L. A dielectric omnidirectional reflector. Science 1998, 282, 1679-1682.

6. Dowling, J.P. Mirror on the wall: You're omnidirectional after all? Science 1998, 282, 1841-1842. 
7. Yablonovitch, E. Engineered omnidirectional external-reflectivity spectra from one-dimensional layered interference filters. Opt. Lett. 1998, 23, 1648-1649.

8. Chigrin, D.N.; Lavrinenko, A.V.; Yarotsky, D.A.; Gaponenko, S.V. All-dielectric one-dimensional periodic structures for total omnidirectional reflection and partial spontaneous emission control. J. Lightw. Technol. 1999, 17, 2018-2024.

9. Lekner, J. Omnidirectional reflection by multilayer dielectric mirrors. J. Opt. A 2000, 2, 349-352.

10. Kramer, B.; MacKinnon, A. Localization: Theory and experiment. Rep. Prog. Phys. 1993, 56, 1469-1564.

11. Segev, M.; Silberberg, Y.; Christodoulides, D.N. Anderson localization of light. Nat. Photonics 2013, 7, $197-204$.

12. Lagendijk, A.; van Tiggelen, B.A. Resonant multiple scattering of light. Phys. Rep. 1996, 270, 143-215.

13. Van Tiggelen, B.A. Transverse Diffusion of Light in Faraday-Active Media. Phys. Rev. Lett. 1995, 75, 422-424.

14. Steurer, W.; Sutter-Widmer, D. Photonic and phononic quasicrystals. J. Phys. D 2007, 40, R229-R247.

15. Poddubny, A.; Ivchenko, E. Photonic quasicrystalline and aperiodic structures. Phys. E 2010, 42, 1871-1895.

16. Dal Negro, L.; Boriskina, S.V. Deterministic aperiodic nanostructures for photonics and plasmonics applications. Laser Photon. Rev. 2012, 6, 178-218.

17. Steinhardt, P.J.; Ostlund, S. The Physics of Quasicrystals; World Scientific: Singapore, 1987.

18. Senechal, M. Quasicrystals and Geometry; Cambridge University Press: Cambridge, UK, 1995.

19. Janot, C. Quasicrystals: A Primer, 2nd ed.; Oxford University Press: Oxford, UK, 2012.

20. Vardeny, Z.V.; Nahata, A.; Agrawal, A. Optics of photonic quasicrystals. Nat. Photonics 2013, 7, $177-187$.

21. Dal Negro, L.; Wang, R.; Pinheiro, F. Structural and Spectral Properties of Deterministic Aperiodic Optical Structures. Crystals 2016, 6, 161.

22. Albuquerque, E.L.; Cottam, M.G. Theory of elementary excitations in quasiperiodic structures. Phys. Rep. 2003, 376, 225-337.

23. Gumbs, G.; Ali, M.K. Dynamical maps, Cantor spectra, and localization for Fibonacci and related quasiperiodic lattices. Phys. Rev. Lett. 1988, 60, 1081-1084.

24. Nori, F.; Rodríguez, J.P. Acoustic and electronic properties of one-dimensional quasicrystals. Phys. Rev. B 1986, 34, 2207-2211.

25. Dulea, M.; Johansson, M.; Riklund, R. Localization of electrons and electromagnetic waves in a deterministic aperiodic system. Phys. Rev. B 1992, 45, 105-114.

26. Boriskina, S.V.; Gopinath, A.; Negro, L.D. Optical gap formation and localization properties of optical modes in deterministic aperiodic photonic structures. Opt. Express 2008, 16, 18813-18826.

27. Maciá, E.; Domínguez-Adame, F. Physical nature of critical wave functions in Fibonacci systems. Phys. Rev. Lett. 1996, 76, 2957-2960.

28. Thiem, S.; Schreiber, M.; Grimm, U. Wave packet dynamics, ergodicity, and localization in quasiperiodic chains. Phys. Rev. B 2009, 80, 214203.

29. Fujiwara, T.; Kohmoto, M.; Tokihiro, T. Multifractal wave functions on a Fibonacci lattice. Phys. Rev. B 1989, $40,7413-7416$.

30. Monsoriu, J.A.; Villatoro, F.R.; Marín, M.J.; Pérez, J.; Monreal, L. Quantum fractal superlattices. Am. J. Phys. 2006, 74, 831-836.

31. Esaki, K.; Sato, M.; Kohmoto, M. Wave propagation through Cantor-set media: Chaos, scaling, and fractal structures. Phys. Rev. E 2009, 79, 056226.

32. Maciá, E. The role of aperiodic order in science and technology. Rep. Prog. Phys. 2006, 69, 397-441.

33. Maciá, E. Aperiodic Structures in Condensed Matter: Fundamentals and Applications; CRC Press: Boca Raton, FL, USA, 2009.

34. Merlin, R.; Bajema, K.; Clarke, R.; Juang, F.Y.; Bhattacharya, P.K. Quasiperiodic GaAs-AlAs Heterostructures. Phys. Rev. Lett. 1985, 55, 1768-1770.

35. Kohmoto, M.; Kadanoff, L.P.; Tang, C. Localization problem in one dimension: Mapping and escape. Phys. Rev. Lett. 1983, 50, 1870-1872.

36. Kohmoto, M.; Sutherland, B.; Iguchi, K. Localization in optics: Quasiperiodic media. Phys. Rev. Lett. 1987, $58,2436-2438$.

37. Liu, N.H. Propagation of light waves in Thue-Morse dielectric multilayers. Phys. Rev. B 1997, 55, $3543-3547$.

38. Tamura, S.; Nori, F. Transmission and frequency spectra of acoustic phonons in Thue-Morse superlattices. Phys. Rev. B 1989, 40, 9790-9801. 
39. Kolář, M.; Ali, M.K.; Nori, F. Generalized Thue-Morse chains and their physical properties. Phys. Rev. B 1991, 43, 1034-1047.

40. Luck, J.M. Cantor spectra and scaling of gap widths in deterministic aperiodic systems. Phys. Rev. B 1989, $39,5834-5849$.

41. Cojocaru, E. Forbidden gaps in finite periodic and quasi-periodic Cantor-like dielectric multilayers at normal incidence. Appl. Opt. 2001, 40, 6319-6326.

42. Holzer, M. Three classes of one-dimensional, two-tile Penrose tilings and the Fibonacci Kronig-Penney model as a generic case. Phys. Rev. B 1988, 38, 1709-1720.

43. Severin, M.; Dulea, M.; Riklund, R. Periodic and quasiperiodic wavefunctions in a class of one-dimensional quasicrystals: An analytical treatment. J. Phys. Condens. Matter 1989, 1, 8851-8858.

44. Dulea, M.; Severin, M.; Riklund, R. Transmission of light through deterministic aperiodic non-Fibonaccian multilayers. Phys. Rev. B 1990, 42, 3680-3689.

45. Oh, G.Y.; Lee, M.H. Band-structural and Fourier-spectral properties of one-dimensional generalized Fibonacci lattices. Phys. Rev. B 1993, 48, 12465-12477.

46. Chakrabarti, A.; Karmakar, S.N. Renormalization-group method for exact Green's functions of self-similar lattices: Application to generalized Fibonacci chains. Phys. Rev. B 1991, 44, 896-899.

47. Fu, X.; Liu, Y.; Zhou, P.; Sritrakool, W. Perfect self-similarity of energy spectra and gap-labeling properties in one-dimensional Fibonacci-class quasilattices. Phys. Rev. B 1997, 55, 2882-2889.

48. Wang, X.; Grimm, U.; Schreiber, M. Trace and antitrace maps for aperiodic sequences: Extensions and applications. Phys. Rev. B 2000, 62, 14020-14031.

49. Chen, Y.; Yang, X.; Guo, Q.; Lan, S. Second-harmonic generation in $\operatorname{GF}(m, 1)$ ferroelectric superlattices. J. Phys. Condens. Matter 2006, 18, 2587-2600.

50. Thiem, S.; Schreiber, M. Photonic properties of metallic-mean quasiperiodic chains. Eur. Phys. J. B 2010, 76, 339-345.

51. Zhang, Z.; Tong, P.; Gong, J.; Li, B. Wave packet dynamics in one-dimensional linear and nonlinear generalized Fibonacci lattices. Phys. Rev. E 2011, 83, 056205.

52. Thiem, S.; Schreiber, M.; Grimm, U. Light transmission through metallic-mean quasiperiodic stacks with oblique incidence. Philos. Mag. 2011, 91, 2801-2810.

53. Costa, C.H.O.; Vasconcelos, M.S. Band gaps and transmission spectra in generalized Fibonacci $\sigma(p, q)$ one-dimensional magnonic quasicrystals. J. Phys. Condens. Matter 2013, 25, 286002.

54. Barriuso, A.G.; Monzón, J.J.; Yonte, T.; Felipe, A.; Sánchez-Soto, L.L. Omnidirectional reflection from generalized Fibonacci quasicrystals. Opt. Express 2013, 21, 30039-30053.

55. Brandão, E.R.; Costa, C.H.; Vasconcelos, M.S.; Anselmo, D.H.A.L.; Mello, V.D. Octonacci photonic quasicrystals. Opt. Mater. 2015, 46, 378-383.

56. Sánchez, F.; Sánchez, V.; Wang, C. Renormalization approach to the electronic localization and transport in macroscopic generalized Fibonacci lattices. J. Noncryst. Solids 2016, 450, 194-208.

57. Spinadel, V.W. The metallic means family and multifractal spectra. Nonlinear Anal. 1999, 36, 721-745.

58. Lloyd, S. Measures of Complexity: A Non-Exhaustive List. Available online: http://web.mit.edu/esd.83/ www /notebook/Complexity.PDF (accessed on 20 April 2017).

59. Aboy, M.; Hornero, R.; Abásolo, D.; Álvarez, D. Interpretation of the Lempel-Ziv Complexity Measure in the Context of Biomedical Signal Analysis. IEEE Trans. Biomed. Eng. 2006, 53, 2282-2288.

60. Lothaire, M. Combinatorics on Words, 2nd ed.; Cambridge University Press: Cambridge, UK, 1997.

61. Queffélec, M. Substitutional Dynamics Systems-Spectral Analysis; Lectures Notes in Mathematics; Springer: Berlin, Germany, 1987; Volume 1294.

62. Dunlap, R.A. The Golden Ratio and Fibonacci Numbers; World Scientific: Singapore, 1997.

63. Sánchez-Soto, L.L.; Monzón, J.J.; Barriuso, A.G.; Cariñena, J.F. The transfer matrix: A geometrical perspective. Phys. Rep. 2012, 513, 191-227.

64. Monzón, J.J.; Yonte, T.; Sánchez-Soto, L.L.; Cariñena, J.F. Geometrical setting for the classification of multilayers. J. Opt. Soc. Am. A 2002, 19, 985-991.

65. Yonte, T.; Monzón, J.J.; Sánchez-Soto, L.L.; Cariñena, J.F.; López-Lacasta, C. Understanding multilayers from a geometrical viewpoint. J. Opt. Soc. Am. A 2002, 19, 603-609.

66. Bombieri, E.; Taylor, J.E. Which distributions of matter diffract? An initial investigation. J. Phys. Colloq. 1986, 47, 19-28.

67. Bombieri, E.; Taylor, J. Quasicrystals, tilings, and algebraic number theory. Contemp. Math. 1987, 64, $241-264$. 
68. Godrèche, C.; Luck, J.M. Indexing the diffraction spectrum of a non-Pisot self-similar structure. Phys. Rev. B 1992, 45, 176-185.

69. Meyer, C.D. Matrix Analysis and Applied Linear Algebra; Society for Industrial and Applied Mathematics: Philadelphia, PA, USA, 2000.

70. Severin, M.; Riklund, R. Using the Fourier spectrum to classify families of generalised extensions of the Fibonaccian lattice. J. Phys. Condens. Matter 1989, 1, 5607-5612.

71. Cheng, Z.; Savit, R. Structure factor of substitutional sequences. J. Stat. Phys. 1990, 60, 383-393.

72. Bellissard, J.; Bovier, A.; Ghez, J.M. Gap labelling theorems for one-dimensional discrete Schrödinger operators. Rev. Math. Phys. 1992, 4, 1-37.

73. Manson, S.M. Simplifying complexity: A review of complexity theory. Geoforum 2001, 32, 405-414.

74. Lempel, A.; Ziv, J. On the complexity of finite sequences. IEEE Trans. Inf. Theory 1976, IT-22, 75-81.

75. Thai, Q. Available online: https://de.mathworks.com/matlabcentral/fileexchange/38211-calc-lz-complexity (accessed on 15 June 2012).

76. Schürmann, T. Scaling behaviour of entropy estimates. J. Phys. A 2002, 35, 1589.

(C) 2017 by the authors. Licensee MDPI, Basel, Switzerland. This article is an open access article distributed under the terms and conditions of the Creative Commons Attribution (CC BY) license (http:// creativecommons.org/licenses/by/4.0/). 